\title{
COMPARATIVE STUDY IN THE DIAGNOSIS OF ANEMIA BY SYSMEX KX-21N HEMATOLOGY ANALYZER WITH PERIPHERAL BLOOD SMEAR
}

\author{
Ejaz Farah, Ahmed Mehwish \& Hassan Ali Nafisa \\ Dow University of health science, \\ Karachi, Pakistan.
}

Corresponding author: farah-bs@hotmail.com

\begin{abstract}
In this era, automated peripheral blood count for the diagnosis of anemia is widely accepted in routine practice. Despite the sophistication of present day instruments, there is still need to depend on manual microscopic scan of peripheral blood smear for the diagnosis and appropriate treatment of different types of anemia. Also, the correlation between automated hematology analyzer and manual microscopic scan is rare and conflicting. Hence, the present study was designed to demonstrate a procedure that, the uses of manual scan is to validate rather than to replace automation. The benefit of manual microscopic scan is the ability to identify clinically significant cell type that are not quantifiable by instruments as it "flags" on such type of cells. Therefore, automated hematology analyzer are as reliable as standard manual scan, even though the latter gives additional diagnostic information through blood pictures and less imprecision and technical error over automation. Patient care and lab operations could be optimized as a validation procedure rather than as a reflexive substitute. Hence manual microscopic scan is still gold standard in the diagnosis and classification of anemia and other blood disorders. Whereas usage of automated method would ease our workload and save time for patients.
\end{abstract}

KEY WORDS

anemia, automated hematology analyzer, peripheral blood smear

\section{INTRODUCTION}

Anemia is common medical problem associated with an increased mortality and morbidity risk and it reduces quality of life (Joosten, 2004; Bhasin, 2011). In 1968 WHO define it, a condition in which hemoglobin contents lowers due to deficiency of essential nutrients (iron, folate and vitamins) as well as the number of red blood cells are insufficient to meet the body's physiologic need(WHO, 2011). It is not a diagnosis itself, but it is a sign of underlying disease. Hence, the evaluation of a patient with anemia is directed at elucidating the causes for the patient's reduced number and morphological changes of red blood cells. In the diagnosis of different anemia, morphological changes of red blood cells provide an important diagnostic value. (Lantis, 2003)

Anemia is better classified into its types on the basis of both qualitative and quantitative results. An initial morphologic classification of anemia with integration of red blood cell indices and morphologic characteristics is probably most useful (Perkins, 1995). Hence it is categorized by cell size as microcytic, normocytic, or macrocytic. Microcytic anemia associated with iron deficiency is the most prevalent micronutrient deficiency disease in the world affecting 2 billion people (James, 2006; Demayer, 1985 \& Stoltzfus, 2001). Though macrocytic anemia associated with $\mathrm{B}_{12}$ and folate deficiency is not very common in Pakistani population as compared to microcytic anemia but it is still has a prevalence in infants. In the diagnosis of different types of anemia morphological changes of red blood cells provide an important diagnostic value. For this reason classification of anemia is also very important for the appropriate treatment of underlying disease. Nowadays, the use of automated hematology analyzer in the diagnosis of anemia has become universal. Most of the analyzers measure the hemoglobin, erythrocyte count, and calculate mean corpuscular hemoglobin (MCH), mean corpuscular volume (MCV) mean corpuscular hemoglobin concentration (MCHC) and hematocrit(Ralph O, 1987). The major disadvantage with automated analyzer is that it uses an array of flags that serve as signals of the potential need for manual review of automated results (Paul, 1965). The manual scan of peripheral blood smear provides additional help for the diagnosis of anemia. The benefit of manual scan is to detect clinically significant morphological abnormalities (pencil cells, sickle cells, tear drop cells, schistocytes e.t.c) that are not quantifiable by the instrument. Therefore the results of hematology analyzer should validate with manual scan of peripheral blood smear and both the results must correlate with each other (Stiene, 1998). In comparison, hematology analyzer gives quantitative results whereas peripheral smear gives qualitative results. Peripheral smear is labor intensive but it is a cost effective and more sensitive method than hematology analyzer. Reduction in the manual scan of peripheral blood smear increases the release of less accurate and it has a negative effect on the diagnosis and treatment of anemia as well (Paul, 1965). In contrast of the manual scan, the hematology analyzer reduces the labor cost but create decision making problem to finalize the diagnosis (Pierre, 2002). In most laboratories, anemia is diagnosed either by hematology analyzer and peripheral smear (Provan, 2000). However hematology analyzer only shows normal or low level of hemoglobin but it is not able to reveal the variation of abnormal cells(Vishwanath, 2001). Hence it is mandatory to scan every slide manually. The aim of this study is to diagnose different type of anemia's affirming the results of automated analyzer with manual scan of peripheral blood smear and to increase the prophylaxis measures by screening the population which may develop anemia in future.

\section{MATERIALS AND METHODS}

The hematology department of Sindh government hospital Karachi performs more than 30,000 complete blood count (CBC) per year. Samples are run on SYSMEX KX-21N hematology analyzer. SYSMEX KX-21N uses an array of flags that serve as signals of the potential need for manual review of automated results. Population of both anemic and healthy individuals in the age group 0-60 years were evaluated for the study.

\section{SAMPLE SIZE}

Total 350 apparently healthy and anemic patients in the age group of 0 60 years were enrolled in this study.

COLLECTION OF BLOOD SAMPLE

$3 \mathrm{ml}$ of blood samples were collected aseptically by standard phlebotomy technique by trained phlebotomist from each subject into tri-potassium Ethylenediamine tetra-acetic acid (K3 EDTA) anticoagulant.

\section{SCREENING OF BLOOD SAMPLES}


Hematological parameters are obtained by using automated hematology cell counter SYSMEX KX-21N. The hemoglobin concentration and red blood cell (RBC) indices, encompassed mean corpuscular volume (MCV), mean corpuscular hemoglobin (MCH), and mean corpuscular hemoglobin concentration (MCHC) and erythrocyte count. SYSMEX KX-21N works on the principle of light deflection method.

\section{RESULTS}

In this prospective study, demographic variables that include such information as total population were 350 individuals apparently healthy and anemic. They were aged between $0-60$ years. Among 350 individuals, males constituted $113(32.3 \%)$, while females were 237
(67.7 \%). Population was analyzed for complete blood count on hematology analyzer and visual examination of peripheral blood smear. In which health status, $(46.8 \%)$ seems to be anemic and healthy individuals are $(53.1 \%)$.On screening of 350 individuals, the concordance between hematology analyzer and visual examination of peripheral blood smear is 278 (78\%). Of the 278 screened cases, $142(40.0 \%)$ are true negative anemia while the remaining $136(38.9 \%)$ are true positive anemia. For these 278 cases, results generated by automated hematology analyzer validated by the scan of peripheral blood smear. Furthermore, both the results of automated analyzer and peripheral smear revealed statistically significant differences (p-value < 0.001).In present study, for the diagnosis of anemia the conflicting findings between quantitative and qualitative analysis is given in [Table 1].

[Table 1]:

No of positive and negative cases of anemia in automation and microscopy

\begin{tabular}{|c|c|c|}
\hline & AUTOMATION & MICROSCOPY \\
\hline \multirow{2}{*}{$\begin{array}{c}\text { Anemia Positive } \\
\text { Anemia Negative }\end{array}$} & 196 & 147 \\
\cline { 2 - 3 } & & 203 \\
\cline { 2 - 3 } & 154 & \\
\hline
\end{tabular}

Of the 350 screened cases, $76(21.7 \%)$ had discordant findings between hematology analyzer and peripheral blood picture. A summary of prospective findings in which the discordance between quantitative and qualitative analysis is given in [Table 2]

[Table 2]:

Important Findings of 76 cases in which the results of automated analyzer and Peripheral smear are not correlated

\begin{tabular}{|c|c|c|c|c|c|}
\hline \multirow[t]{2}{*}{ Cases (n) } & \multicolumn{3}{|c|}{ Analyzer results } & \multirow[t]{2}{*}{ P.Smear results } & \multirow[t]{2}{*}{ Suspected disease } \\
\hline & $\mathrm{Hb}$ & RBC & Indices & & \\
\hline 31 & $\downarrow$ & $\rightarrow$ & $\rightarrow$ & Normocytic,Normochromic & Chronic disease, acute blood loss \\
\hline 20 & $\downarrow$ & $\downarrow$ & $\downarrow$ & Normocytic,Normochromic,Teardrop,Poikilor & Posthemorhegic, Choronic disease \\
\hline 5 & $\rightarrow$ & $\rightarrow$ & $\rightarrow$ & Hypochromic,Normocytic,Poikilocytosis & IDA, Thalessemia \\
\hline 3 & $\downarrow$ & $\uparrow$ & $\downarrow$ & Normocytic,Normochromic & Polycythemia,Thalessemia \\
\hline 2 & $\downarrow$ & $\downarrow$ & $\uparrow$ & Hypochromic,Macrocytic,Poikilocytosis & Folate and B12 defeciency \\
\hline 6 & $\rightarrow$ & $\rightarrow$ & $\rightarrow$ & Hypochromic,Macrocytic,Poikilocytosis & Mild Defeciency of Vit B12 \\
\hline 2 & $\rightarrow$ & $\rightarrow$ & $\rightarrow$ & Hypochoromic,Microcytic,Aniso,Poikilocytos & s IDA,Sideroblastic Anemia \\
\hline 2 & $\downarrow$ & $\downarrow$ & $\downarrow$ & Macrocytic,Hypochoromic & IDA, B12 Defeciency \\
\hline
\end{tabular}

$\boldsymbol{n}$ : Number of cases

Indices: $\mathrm{RBCs}$ Indices include $\mathrm{MCV}, \mathrm{MCH}$ and $\mathrm{MCHC}$

IDA: Iron deficiency anemia

$\rightarrow:$ Normal Value

$\uparrow:$ Increased Value

$\downarrow$ Decreased Value

[Table-2] For 31 of these 72 cases revealed normal morphology of red blood cells but abnormal levels of quantitative results (low levels of hemoglobin),including 20 cases with low levels of hemoglobin, red blood cells, mean corpuscular volume, mean corpuscular hemoglobin and mean corpuscular hemoglobin concentration which should be validated by visual scan of peripheral blood smear. But peripheral blood film shows norma morphologic picture which indicate errors in automated analysis. The discordance was due to presence of agglutinated RBCs, fragmented RBCs or abnormal blood cells which were not detected by automated hematology analyzer. Nevertheless it is necessary to scan every blood film as it is considered microscopy is a gold standard investigation; it can differentiate not only different types of anemia but also hemoglobinopathies and other blood disorders The validation technique of automated hematology analyzer with peripheral smear should be implemented in every lab not for the sake of expediency but to assure performance of test that would be of greatest benefit to patient care. 


\section{DISCUSSION}

The present study assessed the clinical viability of manual scan of peripheral blood smear for the diagnosis of different types of anemia, thalassemia, sickle cell disease, sideroblastic anemia and hemoglobinopathies. Anemia is defined as decrease number of red blood cells or less than the normal quantity of hemoglobin in the blood. Anemia is typically diagnosed on a complete blood count. Apart from reporting the number of red blood cells and the hemoglobin level, the hematology analyzer measure the size of the red blood cells. Examination of a stained blood smear using a microscope can also be helpful, and it is sometimes a necessity in regions of the world where automated analysis is less accessible. Anemia is diagnosed by the results of both hematology analyzer and peripheral blood smear. Automated blood counts are widely accepted in routine screening practice but of its greater acceptance many laboratories still reflexively perform manual scan of peripheral blood smear based solely on abnormal automated results or instruments flags before any triage step (Lantis, 2003; Samuel, 2010).The benefit of manual scan is the ability to identify clinically significant cell types (pencil cells, tear drop cells, burr cells, schistocytes, target cells, sickle cells, blast cells as shown in our study) that are not quantifiable by instruments, but that produce flags on the automated results(Cornbleet, 1997; Dutcher, 1985 \& Gulati, 2001). In current study, we screened up to 350 subjects both healthy and anemic individuals. The direct microscopic visualization of peripheral blood film revealed among the 350 subjects, 147 subjects were anemic, among 203 subjects; 127 subjects were healthy while 76 subjects revealed other blood disorders.Table-1. These findings are very important in the diagnosis of various blood disorders that are not evaluated by automation. However, the discrepancy between automation and manual scan of peripheral blood in the measurement of hemoglobin and red blood count may result in misclassification for the diagnosis of anemia. This signified that the manual microscopic method still has some advantages over the automated method (Samuel, 2010; Hyun, Gulati, 1991; Takubo, 1990 \& Kamentsky, 1996). Also, this is in agreement with an earlier report by Takubo and Tatsuni (Takubo, 1990) who reported discrepancies in a quality control (QC) survey. In addition our study revealed statistically 273 subjects out of 350 , correlated positively when both automated and manual methods were compared. This manifests that the automated analyzer (Sysmex KX-21N) readings correlated well with the manual methods. This emphasized by an earlier report by Atilola and Mac Karthy et al (Atilola \& Kamentsky, 1996, McCarthy, Capuilari \& spellary, 2005) . The results of the present study are in contrast with an earlier report by Pierre and Novis et al.(Pierre, 2002, Novis et.al 2006) who reported that automated hematology analyzer are more accurate in the detection of specimens with morphological abnormality than the traditional eye count method. Whereas, [Table -2] among 350 subjects, 76 would have discordance findings and contributed to some statistical variations observed between hematology analyzer and manual scan of peripheral blood smear adopted in our study. De Gruchy, also affirmed that the manual scan of peripheral blood smear which may provide important diagnostic help in patients with normal blood counts are frequently overlooked, his results illustrated that the four non-anemic patients reported had normal blood counts. In a blood smear of each he found important morphological abnormalities. In each case the findings came as a surprise to the clinician and led to a diagnosis which he had not formerly suspected(Joosten E, 2004). In most automated laboratories, manual microscopic examination are essential to the cases for which instrument "flags", indicate the potential presence of cells not reliably identifiable by automated methods or findings that may interfere with automated analysis (such as overlap in the distribution of different cell types or interference from matrix components such as cryoglobulin) (Pierre, 2002, Paul G \& Dorothy, 1965) Similarly, Lantis et al. (Lantis et.al
2003) and LKE et al. (Samuel et.al 2010) also support our study and suggested that microscopic examination should always be used to validate the automated results. In Lantis study, for 12 of these 15 cases, the discordance was due to the presence of $3 \%$ or more metamyelocytes and/or myelocytes, including 11 cases with $5 \%$ or fewer metamyelocytes and/or myelocytes and 1 with $8 \%$ metamyelocytes(Lantis et,al 2003). In Samuel O LKE study, the blood film report by the manual method showed that $50 \%$ of the subjects were normocytic, normochromic while the other $50 \%$ revealed different abnormal blood pictures. Also, there were statistically significant differences $(\mathrm{p}<0.05)$ in mean cell hemoglobin concentrations (MCHC) between the two methods (Samuel, 2010). In recent study, most of the cases have unremarkable cell counts and red cell indices by automation; this inaccuracy indicates whether the presence of abnormal cells, blast cells or other technical errors, these results needed to be validated manually by traditional eye count method.The approach of current study is to diagnose different type of anemia and other blood disorders by manual scan of peripheral blood smear, so the appropriate treatment can be given on time. We adopted attempts to optimally apply both automated and manual scan to maximize the clinical value added to the patient care after manual scan because it can differentiate different types of anemia and other blood disorders as well(Lantis et.al 2003). This will rule out proper and appropriate treatment and patient care. From the therapeutic stand point not only it is useful to know the cause of anemia, but it is important to differentiate the anemia in accordance with the type of disorder in the hematopoietic system (Gulati, 2001 ) Review criteria for flagged results must be designed to optimize the value added to patient care, including the judicious appropriation of time, money, and skills in the laboratory. There is no intrinsic value added to patient care by the reflexive performance based on instrument flags (Lantis, 2003). Hence our study discovered that the microscopic examination of peripheral blood smear is gold standard in the diagnosis of various types of anemia. By the help of manual microscopic scan we can avoid misdiagnosis and non-rejection mortality rates. Many would add that the skillful examination of a well prepared blood smear is one of the most specific examinations in nonanemic patients as well (Paul, 1965). The leading advantage of recent study is the patient care and laboratory operations can be optimized by using manual microscopic examination as a validation procedure rather than as a reflexive substitute for automated methods. There is no clinical rationale for reflex performance of manual microscopic scan based solely on instrument warnings. Usage of automated method would ease our workload and save time for patients but it must be validate by manual scan(Lantiset, 2003; Samuel, 2010)

\section{CONCLUSION}

The automated hematology analyzer readings are as reliable as the standard manual method, even though the manual scan of peripheral smear provides additional diagnostic information. The result of the study shows that the automated hematology analyzer are appropriate for screening purposes because it increases the turnaround time and reduces the labor cost. But to diagnose and differentiate different types of anemia manual scan of peripheral smear is a method of choice. Clinical laboratories can optimize patient care by using manual methods for validation, rather than replacement of automated method. There is no clinical rationale for reflex performance of manual microscopy based solely on quantitative or qualitative abnormalities reported by automated method. Hence microscopic examination of blood should always be used to validate the automated method, as previously suggested by LANTIS. et al and LKE. et al.Patients care and lab operators should be optimized by using microscopic examination in conjunction with automated methods, especially in the 
diagnosis of different types of anemia and their appropriate treatment.

\section{REFERENCES}

- Joosten, E. (2004). Strategies for the laboratory diagnosis of some common causes of anaemia in elderly patients. Gerontology, 50(2), 49-56.

- Bhasin, A., \& Medha, Y. R. (2011). Characteristics of Anemia in Elderly. Indian J Hematol Blood Transfusion, 27(1), 26-32.

- $\quad$ Lantis, K. L., Harris, R. J., Davis, G., Renner, N., \& Finn, W. G. (2003). Elimination of Instrument-Driven Reflex Manual Differential Leukocyte Counts Optimization of Manual Blood Smear Review Criteria in a High-Volume Automated Hematology Laboratory. American journal of clinical pathology, 119(5), 656-662.

- Perkins and Sherrie (1995). Practical diagnosis of hematologic disorders. Chicago, ASCP press.

- Rebecca, J.S. (2001). Iron-Deficiency Anemia: Reexamining the Nature and Magnitude of the Public Health Problem. American society for nutritional sciences, J. Nutr. (131). 565-567.

- WHO, (2011) Vitamin and Mineral Nutrition information system. Hemoglobin Concentration for the diagnosis of anemia and assessment of severity. WHO/NMH/NHD/MNM/11.1.

- McClung, J. P., Marchitelli, L. J., Friedl, K. E., \& Young, A. J. (2006). Prevalence of iron deficiency and iron deficiency anemia among three populations of female military personnel in the US Army. Journal of the American college of nutrition, 25(1), 64-69.

- DeMaeyer, E. M., \& Adiels-Tegman, M. (1985). The prevalence of anaemia in the world. World health statistics quarterly. Rapport trimestriel de statistiques sanitaires mondiales, 38(3), 302 .

- $\quad$ Stoltzfus, R. J. (2001). Defining iron-deficiency anemia in public health terms: a time for reflection. The Journal of nutrition, 131(2), 565S-567S

- Wallerstein Jr, R. O. (1987). Laboratory evaluation of anemia. Western Journal of Medicine, 146(4), 443.

- Hattersley, P. G., \& Ragusa, D. (1965). Don't Forget Morphology - The Importance of Evaluation of Blood Smears. California medicine, 103(3), 175.

- Stiene-Martin, E. A., Lotspeich-Steininger, C. A., \& Koepke, J. A. (1998). Clinical hematology: principles, procedures, correlations. Lippincott Williams \& Wilkins.

- Pierre, R.V. (2002). The demise of the eye count leucocyte differential. Clin Lab Med 22(1), 279-297.

- Provan, D., and Weeatherall., D (2000) Red cells Acquired anemia and polycythemia. Lancet, 355:1260.

- Viswanath, D., Hegde, R., Murthy, V., Nagashree, S., \& Shah, R. (2001). Red cell distribution width in the diagnosis of iron deficiency anemia. The Indian Journal of Pediatrics, 68(12), 1117-1119.

- $\quad$ Ike, S. O., Nubila, T., Ukaejiofo, E. O., Nubila, I. N., Shu, E. N., \& Ezema, I. (2010). Comparison of haematological parameters determined by the Sysmex KX-2IN automated haematology analyzer and the manual counts. BMC clinical pathology, 10(1), 3.

- Novis, D. A., Walsh, M., Wilkinson, D., St. Louis, M., \& Ben-Ezra, J. (2006). Laboratory productivity and the rate of manual peripheral blood smear review: a College of American Pathologists Q-Probes study of 95141 complete blood count determinations performed in 263 institutions. Archives of pathology \& laboratory medicine, 130(5), 596-601.

- Hanson, C. A. (2002). Peripheral blood and bone marrow: morphology, counts and differentials, and reactive disorders. Clinical Laboratory Medicine, 2nd edn. Williams \& Wilkins, Philadelphia, 797-829.

- Gulati, G.L., Alomari, M., Kochar, W. (2002). Criteria for blood smear review. Lab Med, (33), 374-377.

- Gulati, G. L., Hyun, B. H., \& Ashton, J. K. (1992). Advances of the past decade in automated hematology. American journal of clinical pathology, 98(4 Suppl 1), S11.

- Hyun, B. H., Gulati, G. L., \& Ashton, J. K. (1991). Differential leukocyte count: manual or automated, what should it be?. Yonsei Med J, 32(4), 283-291.

- Takubo, T., \& Tatsumi, N. (1999). Quality control in a manual and an automated leukocyte differential count. The Southeast Asian journal of tropical medicine and public health, 30, 66 .

- Atilola, L.R., and Kamentsky, L.A. (1996) Routine ential leucocyte count. Clinical Laboratory Medicine, (15), 289-291.

- McCarthy, J. M., Capullari, T., \& Spellacy, W. N. (2005). The correlation between automated hematology and manually read smears for the determination of nucleated red blood cells in umbilical cord blood. Journal of Maternal-Fetal and Neonatal Medicine, 17(3), 199-201

- Hoyer, J.D. (1993). Leukocyte differential. Mayo Clin Proc, (68), 1027-1028.

- Cornbleet, J., Fernandes, B. J., \& Miers, M. (1997). Streamline your automated hematology laboratory. Roundtable discussion. MLO: medical laboratory observer, 29(2), 43-46.

- Dutcher, T.F. (1985). Automated differentials: a strategy. Blood Cells, 11(1), 49-59.

- Gulati, G. L., Kocher, W., Schwarting, R., Hyland, L. J., Issa, A., Arwood, R., \& Dhanjal, M. (2001). An Assessment of the Coulter Gen - S Automated Flagging System. Lab Medicine, 32(6), 310-317.

- Gulati, G. L., \& Hyun, B. H. (1994). The automated CBC. A current perspective. Hematology/oncology clinics of North America, 8(4), 593.

- Wintrobe, M. M. (1934). Anemia: classification and treatment on the basis of differences in the average volume and hemoglobin content of the red corpuscles. Archives of Internal Medicine, 54(2), 256. 